\title{
Clinical Management of Risk of Radiation Pneumonia with Serum Markers During the Radiotherapy for Patients with Thoracic Malignant Tumors
}

This article was published in the following Dove Press journal: Cancer Management and Research

\author{
Yuxia Deng' \\ Ting Qiu' \\ Nishant Patel ${ }^{\prime}$ \\ Shi Zhou' \\ Tao Xue ${ }^{2}$ \\ Haijun Zhang (iD ${ }^{1,3}$ \\ 'Department of Oncology, Zhongda \\ Hospital, Medical School of Southeast \\ University, Nanjing, Jiangsu, People's \\ Republic of China; ${ }^{2}$ Department of \\ Cardiothoracic Surgery, Zhongda \\ Hospital, Medical School of Southeast \\ University, Nanjing, Jiangsu, People's \\ Republic of China; ${ }^{3}$ Precision Medical \\ Center, Zhongda Hospital, Medical \\ School of Southeast University, Nanjing, \\ jiangsu, People's Republic of China
}

Purpose: Risk of radiation pneumonia (RP) could not be effectively detected due to nonspecific clinical symptoms in the early stage. The purpose of this investigation was to evaluate serum biomarkers of cytokines interleukin-6 (IL-6), C-reactive protein (CRP) and procalcitonin (PCT) for its early detection in patients with thoracic malignant tumors receiving radiotherapy. Patients and methods: The clinical data of 105 patients with thoracic malignant tumors (lung cancer, esophageal carcinoma and mediastinal tumors) treated by radiotherapy were retrospectively analyzed. The patients were divided into RP group and non-RP group according to the Common Terminology Criteria for Adverse Events (CTCAE 5.0). The serum level of IL-6 was detected by chemiluminescence, and the level of CRP was measured by nephelometry during radiotherapy. The level of PCT, one of the specific indicators to distinguish infection and non-infectious etiologies, was also detected by chemiluminescence. Results: Among 105 patients treated by radiotherapy, 28 developed RP, and the other 77 had no RP. There was no significant difference in the risk of RP between patients' factors (age, sex, PS score, smoking, tumor type) and treatment factors (chemotherapy, $\mathrm{V}_{5}$, GTV dose). However, chronic obstructive pulmonary disease (COPD), $\mathrm{V}_{20}$ and mean lung dose (MLD) were significantly different between the two groups $\left(\chi^{2}=4.131,3.986,7.830, \mathrm{P}<0.05\right)$. Furthermore, PCT levels were also found to have insignificant differences between RP group and non-RP group $(P>0.05)$. However, there were significant differences between the groups in the levels of IL- 6 and CRP $(P<$ 0.05). The IL-6 levels significantly increased earlier than that of conventional CT imaging when patients suffering from RP and peaked at 6 weeks during radiotherapy. CRP had a similar change as IL-6. Single cytokine and combination of IL- 6 and CRP possessed a good ability to predict RP with the AUC of IL-6 of $0.89 \pm 0.04(95 \%$ CI, $0.80-0.95, \mathrm{P}<0.001)$, CRP of $0.87 \pm 0.05(95 \% \mathrm{CI}$, 0.78-0.94, P<0.001), IL-6 + CRP of $0.92 \pm 0.03$ (95\% CI, 0.83-0.97, P $<0.001)$, respectively.

Conclusion: The combined detection of serum IL-6, CRP and PCT may be an effectual method for early detection and clinical practice management of risk of RP.

Keywords: cancer, radiotherapy, radiation pneumonitis, interleukin-6, C-reactive protein, procalcitonin, biomarkers

\section{Introduction}

Radiation therapy is one of the main methods for the treatment of thoracic tumors. However, radiation pneumonia (RP) is one of the common complications, leading to respiratory distress and even death in severe cases. ${ }^{1-4} \mathrm{RP}$ could limit the escalation of radiation dose necessary to achieve curative effects meanwhile reducing the quality of life for patients. ${ }^{5,6}$ 
Currently, risk assessment of RP typically depends on physical dosimetric parameters resulting from a population of patients, such as mean lung dose (MLD) and percentage of lung volume exposed to doses exceeding a threshold, which is limited for individual patients. ${ }^{7-11}$ As inflammatory cytokines play important roles in the molecular pathogenesis of lung damage, ${ }^{5,6,12,13}$ it is a perfect outlet for solving the problem through identifying them as biomarkers to more accurately assess individual patient's risk of developing RP.

Multiple cytokines regulate RP through different pathways, which is an inflammatory factor-mediated acute lymphocytic alveolitis. ${ }^{13}$ However, at the present time, there are no reliable and validated predictive assays for the early prediction of RP in the clinical process. The elevation of plasma cytokines interleukin-6 (IL-6) level is positively correlated with the increase of radiation-related lung injury, ${ }^{1,14}$ which could predict acute radiation-induced lung injury ( $\geq$ grade 2$).{ }^{12} \mathrm{C}$-reactive protein (CRP) is an acute-phase reaction protein, which is synthesized and secreted by the liver under the action of IL- 6 and other inflammatory factors. ${ }^{15,16} \mathrm{CRP}$ is a non-specific but sensitive inflammatory biomarker. Early non-specific clinical symptoms are easily confused with intrapulmonary infection, causing the failure to detect early radiation pneumonitis. Procalcitonin (PCT) is a glycoprotein secreted by thyroid C cells, which is one of the specific indicators to distinguish infection and noninfectious etiologies. ${ }^{17-19}$ PCT may have the ability to identify infectious or non-infectious causes to some extent. ${ }^{20}$

In this study, we analyzed the changes of the above serum levels of IL-6, CRP and PCT during the radiotherapy for patients with thoracic malignant tumors. The clinical biomarkers of the risk of RP were identified, thus RP could be diagnosed in the early stage and treated in time independent of conventional computed tomography (CT) image. Our goal is to develop non-invasive and nonimaging biomarkers for early detecting RP even before symptoms occur to avoid the occurrence of pulmonary insufficiency and acute respiratory distress syndrome (ARDS) in RP patients.

\section{Patients and Methods}

\section{Study Population}

Patients with thoracic malignant tumors (lung cancer, esophageal carcinoma and mediastinal tumor) confirmed by histopathology or cytology from October 2013 to November 2017 and treated with three-dimensional conformal radiotherapy or intensity-modulated radiotherapy in Zhongda Hospital,
Medical School of Southeast University were selected for this study. The inclusion criteria were as follows: patients with thoracic malignant tumors receiving radiotherapy had an expected survival of more than 6 months and a PS score $\leq 3$. The exclusion criteria were as follows: patients with stage IV extensive metastasis, left/right pneumonectomy, severe chronic bronchitis, emphysema, corpulmonale or other serious diseases (such as myocardial infarction within 6 months). All patients signed informed consent. The study was approved by the ethics committee of Zhongda Hospital, Southeast University.

\section{Treatment Regimen of Radiotherapy}

All patients underwent routine segmented threedimensional conformal or intensity-modulated radiotherapy with 6MV-X-ray Primus-m linear accelerator. Gross tumor volume (GTV), clinical volume (CTV) and planned target volume (PTV) were delineated according to the definitions of ICRU 50 and 62 reports. There were 5 to 7 fields in total. The shape of irradiation field was designed by beams eye views (BEV), which requires a $95 \%$ isodose line to cover PTV. The total dose of radiotherapy was 40-66 Gy (Median: 54 Gy).

\section{Cytokine Detection}

Blood samples were collected during the radiotherapy for patients with thoracic malignant tumors at different time points of 0 (the beginning of the treatment), 2, 4, 6, 10 weeks. The blood was then centrifuged at $1000 \mathrm{rpm}$ for 15 mins at $4{ }^{\circ} \mathrm{C}$, and the separated serum was stored at $-20^{\circ} \mathrm{C}$ for analysis. The serum IL- 6 and PCT were detected by electrochemiluminescence with the analyzer of COBAS 8000 produced by ROCHE (Switzerland) and the company's kit. The level of CRP in serum was detected by nephelometry with the analyzer of SISTEMA BN II produced by SIEMENS Company and the company's reagent kit. All procedures were carried out strictly according to the kit instructions.

\section{Evaluation of RP}

Patients were evaluated routinely during radiotherapy by physical examination as well as chest CT. During followup, conventional chest $\mathrm{CT}$ scans were performed 1, 3 and 6 months after radiotherapy. Chest $\mathrm{CT}$ should be performed to confirm suspicious symptoms of radiation pneumonia (such as severe cough, elevated body temperature, chest tightness or dyspnea) during or at any time after radiotherapy. RP was graded according to Common Terminology Criteria for Adverse Events (CTCAE, version 5.0) as follows: Grade 1, 
asymptomatic: clinical or diagnostic observations only or intervention not indicated; Grade 2, symptomatic: medical intervention indicated or limited activities of daily living; Grade 3, severe symptoms: limiting self-care for daily living activities or oxygen indicated; Grade 4, life-threatening respiratory compromise: urgent intervention indicated (tracheotomy or intubation); Grade 5, fatal. RP is diagnosed by CTCAE 5.0 evaluation criteria as follows: continuous cough and/or dyspnea occur in patients with radiation pneumonia of grade $(\geq 2)$ and need to be treated clinically. Excluding the above symptoms is caused by the following factors: tumor progression, pulmonary infection (bacteria, fungi or viruses), acute exacerbation of COPD, cardiogenic disease, pulmonary infarction, anemia, drug-induced pneumonia. The changes of CT imaging were mainly spotted shadow, ventilation bronchi sign, strip shadow, lung consolidation shadow or honeycomb in the irradiation area. In addition to the changes in the irradiation area, a few patients also had corresponding imaging changes outside the radiation area. According to whether radiation pneumonia occurred or not, patients were then divided into the radiation pneumonia group (RP group) and non-radiation pneumonia group (no-RP group). Data were not collected and analysed after systemic corticosteroids therapy in RP patients.

\section{Statistical Analysis}

SPSS 22.0 was performed to analyze the data. The Chisquare test was used for categorical variables. Numerical variables conformed to normal distribution through Kolmogorov-Smirnov test were compared using student's T-test. Data of PCT, IL-6 and CRP at each detection point were firstly conformed to the normal distribution, then independent sample $T$-test was used to analyze the data. The prediction parameters of an ROC curve were analyzed by MedCalc software. The difference was statistically significant with $P<0.05$.

\section{Results}

\section{Patients Characteristics and RP Incidence}

The characteristics of the 105 patients included in the retrospective study are listed in Table 1 . Males and females were 77 and 28, respectively, and the average median age was $63.73 \pm 11.78$ years. As far as pathological classification been concerned, there were 49 cases of esophageal carcinomas, 41 cases of lung cancers and 15 cases of mediastinal tumors. Among particular grades of CTCAE 5.0 evaluation
Table I Patients Characteristics $(n=105)$

\begin{tabular}{|c|c|c|c|c|c|}
\hline Item & Sum & $\mathbf{R P}$ & Non-RP & $\chi^{2}$ & $P$ Value \\
\hline Gender & & & & 0.536 & 0.464 \\
\hline Male & 77 & 22 & 55 & & \\
\hline Female & 28 & 6 & 22 & & \\
\hline Age & & & & 1.601 & 0.206 \\
\hline$<65$ years old & 52 & II & 41 & & \\
\hline$\geq 65$ years old & 53 & 17 & 36 & & \\
\hline PS & & & & 1.228 & 0.268 \\
\hline $0-1$ & 86 & 21 & 65 & & \\
\hline $2-3$ & 19 & 7 & 12 & & \\
\hline Smoking & & & & 0.057 & 0.811 \\
\hline Yes & 43 & 12 & 31 & & \\
\hline No & 62 & 16 & 46 & & \\
\hline COPD & & & & 4.131 & 0.042 \\
\hline Yes & 13 & 7 & 6 & & \\
\hline No & 92 & 21 & 71 & & \\
\hline Chemotherapy & & & & 1.193 & 0.275 \\
\hline Yes & 70 & 21 & 49 & & \\
\hline No & 35 & 7 & 28 & & \\
\hline Lung $\mathrm{V}_{20}$ & & & & 3.986 & 0.046 \\
\hline$<25 \%$ & 72 & 15 & 57 & & \\
\hline$\geq 25 \%$ & 33 & 13 & 20 & & \\
\hline Lung $V_{5}$ & & & & 0.992 & 0.319 \\
\hline$<65 \%$ & 82 & 20 & 62 & & \\
\hline$\geq 65 \%$ & 23 & 8 & 15 & & \\
\hline MLD (Gy) & & & & 7.830 & 0.005 \\
\hline$<13$ & 71 & 13 & 58 & & \\
\hline$\geq 13$ & 34 & 15 & 19 & & \\
\hline GTV dose(Gy) & & & & 0.178 & 0.673 \\
\hline$<54$ & 41 & 10 & 31 & & \\
\hline$\geq 54$ & 64 & 18 & 46 & & \\
\hline Tumor type & & & & 0.483 & 0.785 \\
\hline Esophageal & 49 & 13 & 36 & & \\
\hline Lung cancer & 41 & 12 & 29 & & \\
\hline Mediastinum tumor & 15 & 3 & 12 & & \\
\hline
\end{tabular}

Abbreviations: RP, radiation pneumonia; COPD, chronic obstructive pulmonary disease; MLD, mean lung dose; GTV, Gross tumor volume.

criteria, grade 2 , grade 3 , grade 4 and grade 5 were observed $17,7,3$, and 1 number of case, respectively, in the study, thus RP is diagnosed and the incidence of RP was $26.7 \%$. According to the time of the occurrence of RP, there were 2 cases in 4 weeks of radiotherapy, 11 cases in 6 weeks, 14 cases in 10 weeks and 1 case in 18 weeks. The occurrence time of RP was $57.79 \pm 20.88$ days. The onset time of early symptoms was $54.46 \pm 20.89$ days and the abnormal time of CT scan was $59.14 \pm 21.43$ days. The clinical data and 
dosimetric parameters of patients of RP group and non-RP group are compared in Table 1 . There was no significant difference in the risk of RP between patients' factors (age, sex, PS score, smoking, tumor type) and treatment factors (chemotherapy, $\mathrm{V}_{5}$, GTV dose (Gy)). However, chronic obstructive pulmonary disease (COPD), $\mathrm{V}_{20}$ and MLD were significantly different between the two groups $\left(\chi^{2}=4.131\right.$, 3.986, 7.830, $\mathrm{P}<0.05)$.

\section{Changes in the Levels of IL-6 and CRP During the Radiotherapy}

The level of IL-6 increased in patients who developed RP, while it persisted at a lower level in patients who did not develop RP during the radiotherapy. As shown in Figure 1A, there is a statistically significant difference in the level of IL-6 between RP group and non-RP group $(P<0.05)$. What's more, Figure 1A also illustrated that the level of IL-6 in patients with RP started to rise in 2 weeks, which is earlier than that of conventional CT imaging performed to confirm suspicious symptoms of RP (4 weeks). Not come singly but in pairs, in this study, a similar change happened in the levels of CRP, too. Results demonstrated that the levels of CRP in RP group were significantly higher than those in non-RP group, which was shown in Figure 1B.

\section{The Levels of PCT During the Radiotherapy}

Different from the level of IL-6 and CRP, the level of PCT remained consistent throughout the course of radiotherapy and follow-up among patients who did and did not develop
RP. As shown in Figure 2, serum PCT concentrations in both groups were lower than $0.15 \mathrm{ng} / \mathrm{mL}$.

\section{Predictive Risk Assessment Models}

In order to predict the risk of RP, we generate an unbiased cross-validation receiver operating characteristic curve (ROC curve) based on the quantitative level of single and multiple cytokine signals. The threshold of IL-6 and CRP were $14.07 \mathrm{pg} / \mathrm{mL}$ and $32.8 \mathrm{mg} / \mathrm{L}$, respectively. The AUC of IL-6 and CRP were $0.89 \pm 0.04$ (95\% CI, $0.80-0.95, P<0.001)$ and $0.87 \pm 0.05 \quad(95 \% \quad \mathrm{CI}, \quad 0.78-0.94, \quad P<0.001)$ respectively (Figure 3). When combined IL-6 and CRP, the AUC could reach up to $0.92 \pm 0.03$ (95\% $\mathrm{CI}, 0.83-0.97, P<0.001)$.

\section{Comparison of Cytokines and CT in Patients Developed RP}

The chest CT imaging data of $28 \mathrm{RP}$ patients were collected in this study. Early RP had only non-specific symptoms such as cough and chest tightness. In addition, the CT imaging specificity changes (small ground-glass opacity scattered in the irradiation field or long strip, large patch density enhancement shadow) were significantly delayed. ${ }^{21}$ However, serum levels of cytokines IL-6 and CRP increased significantly before the occurrence of radiopneumonitis-specific imaging, and the change of cytokines was $4.68 \pm 2.87$ days earlier than that of RP-specific imaging. In our study, a typical RP case conveyed that cytokines IL-6 and CRP could increase significantly 5 days before obvious clinical symptoms and 12 days before RP characteristic images.

Figure 4 shows a typical case, comparing cytokines and CT in patients developed RP. In this case, an 80-year-old

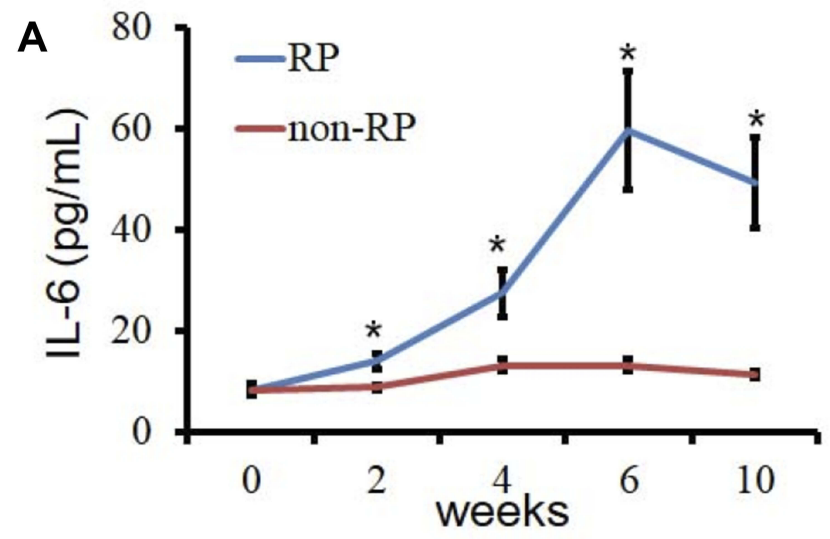

Figure I Changes in the levels of IL-6 during the radiotherapy. (A) the serum level of IL-6 between RP group and non-RP group ( $*$ < 0.05$)$; (B) the serum level of CRP between RP group and non-RP group $(* P<0.05)$.

Abbreviations: RP, radiation pneumonia; IL-6, interleukin-6; CRP, C-reactive protein. 


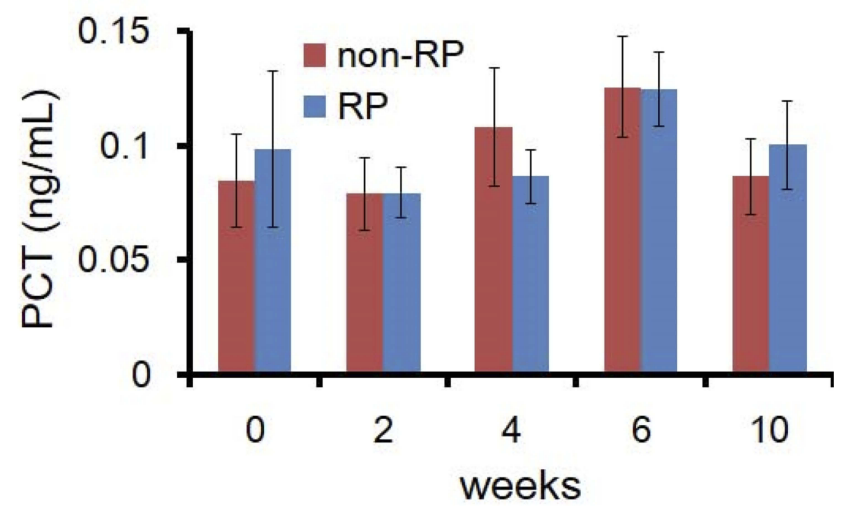

Figure 2 The levels of PCT during the radiotherapy. There is no statistically significant difference in the level of PCT between RP group and non-RP group $(P>0.05)$.

Abbreviations: RP, radiation pneumonia; $\mathrm{PCT}$, procalcitonin.

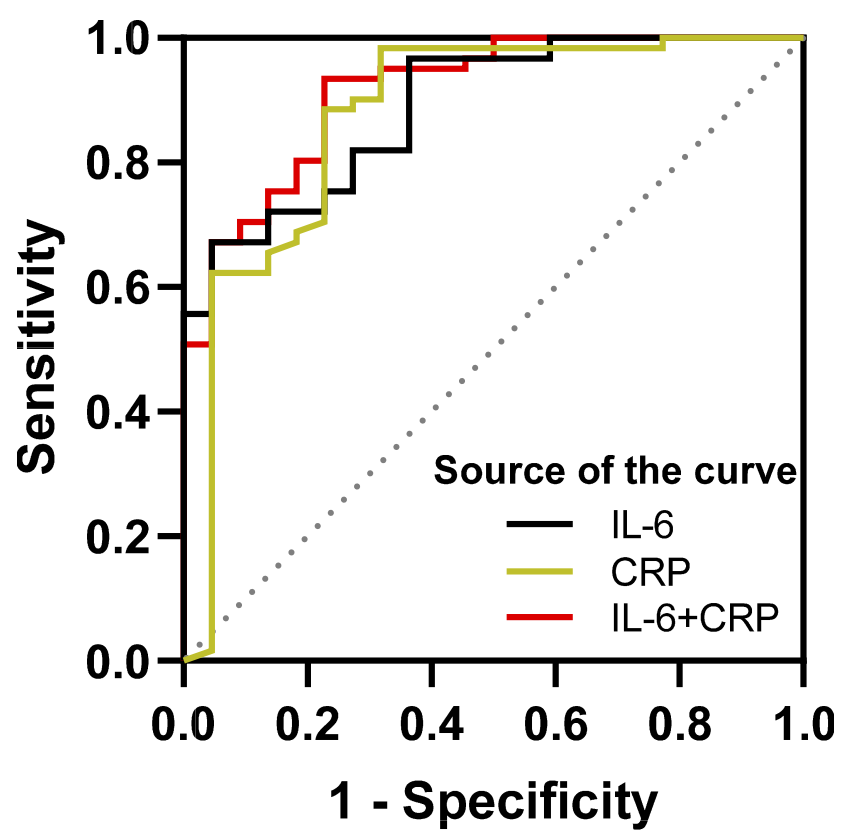

Figure 3 ROC curve based on the sensitivity and specificity of IL-6 alone, CRP alone, and the combination of IL-6 and CRP to predict RP.

Abbreviations: ROC curve, receiver operating characteristic curve; IL-6, interleukin-6; CRP, C-reactive protein; PCT, procalcitonin.

male patient with small cell lung cancer received two courses of Etoposide + Carboplatin regimen. Then, radiotherapy for right lower lobe tumors and mediastinal lymph node metastases began from June 27, 2016 to August 3, 2016. The cumulative dose of radiotherapy was $50 \mathrm{~Gy} / 25 \mathrm{f}$. One month after the end of radiotherapy (10 weeks), he developed right chest pain and was re-examined on September 7, 2016. The patient was generally in good condition with an occasional cough. CT images showed mild interstitial changes in the left lung (Figure 4A-C) with PCT of $0.112 \mathrm{ng} / \mathrm{mL}$, IL-6 of 65.6 $\mathrm{pg} / \mathrm{mL}$ and CRP of $29.8 \mathrm{mg} / \mathrm{L}$. High fever accompanied by chest tightness and asthma occurred in patients on September 12, 2016. At this moment, CT images showed interstitial changes in both lungs (Figure 4D-F), and the range was significantly larger than that on September 7, 2016 with PCT of $0.131 \mathrm{ng} / \mathrm{mL}$, IL-6 of $64.37 \mathrm{pg} / \mathrm{mL}$ and $\mathrm{CRP}$ of $122 \mathrm{mg} / \mathrm{L}$. The patient was diagnosed as RP with grade 3 and received steroid pulse therapy. After 1 week of the steroid-pulse therapy (September 19, 2016), the patient's chest tightness and asthma improved, without fever. CT images suggest interstitial changes in both lungs (Figure 4G-I), which correspond to the characteristic imaging changes of RP with PCT 0.127 of $\mathrm{ng} / \mathrm{mL}$, IL-6 of 105.2 $\mathrm{pg} / \mathrm{mL}$, and CRP $41.9 \mathrm{mg} / \mathrm{L}$. In brief, serum levels of cytokines IL-6 and CRP changed even earlier than radiopneumonia-specific imaging changes while RP developed.

\section{Discussion}

$\mathrm{RP}$ is an inflammation of normal lung tissue caused by radiation therapy and dose-limiting toxicity of thoracic radiotherapy. ${ }^{2}$ Early diagnosis is essential for rapid and adequate treatment to reduce mortality. Biomarker is a promising approach to predict the risk of RP during the radiotherapy for patients with thoracic malignant tumors.

In our study, the levels of IL-6 and CRP in RP group were significantly higher than those in non-RP group. The plasma level of IL- 6 was the highest at 6 weeks during radiotherapy. Furthermore, our data indicate that the changes in serum cytokines IL-6 and CRP are earlier than the characteristic imaging changes of RP. A combination of the two cytokines could better predict the occurrence of RP before the onset of symptoms. Clinical studies have shown that IL-6 may be a biological predictor of lung injury after radiotherapy for thoracic malignant tumors. Rancati et al observed the level of IL-6 in bronchoalveolar lavage fluid of patients with lung cancer after radiotherapy, and $\mathrm{Fu}$ et al found that the level of IL-6 in the lung of the irradiated side increased. ${ }^{9,22}$ RTOG91-03 demonstrated that elevated serum IL-6 levels after 10 Gy lung irradiation could predict grade $\geqq 2$ acute pulmonary toxicity. $^{12}$ The results of Sakai et al indicated that IL-6 knockout mice have an inherent resistance to acute alveolar injury after thoracic vertebral irradiation. ${ }^{23}$ Contrary to our findings, most researchers believe that IL-6 has limited ability to predict RP. ${ }^{6,24,25}$ It may be due to they did not detect plasma levels of IL-6 before the onset of early RP symptoms. CRP is an acute-phase protein, ${ }^{26}$ mainly stimulated by IL-6. ${ }^{15,16}$ Our observations show that the plasma CRP concentration in RP patients also increased significantly with the increase of plasma IL-6 concentration. Because RP is 


\section{September 7, 2016}
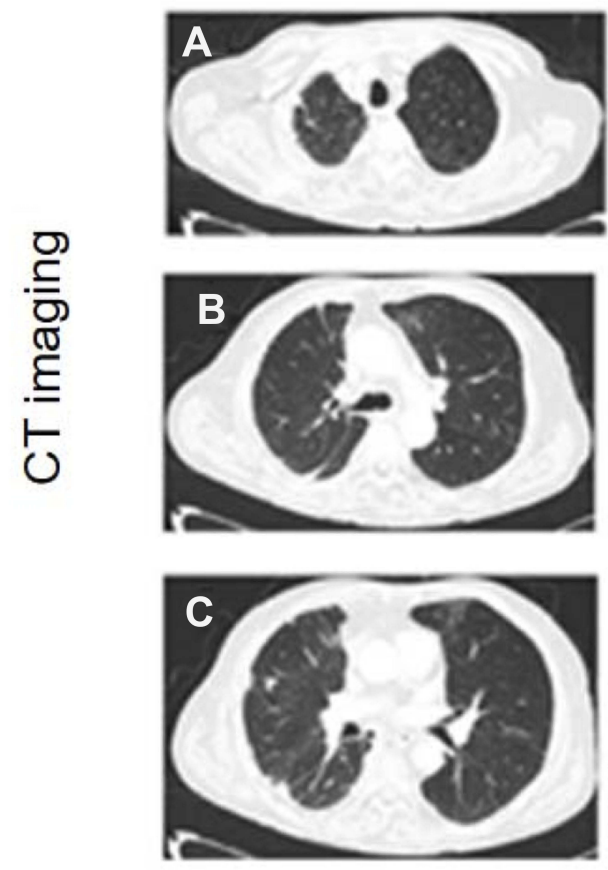

September 12, 2016
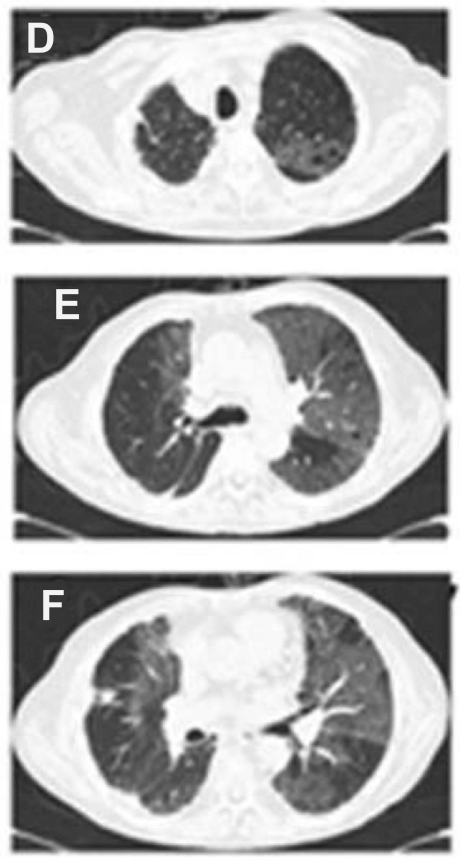

September 19, 2016
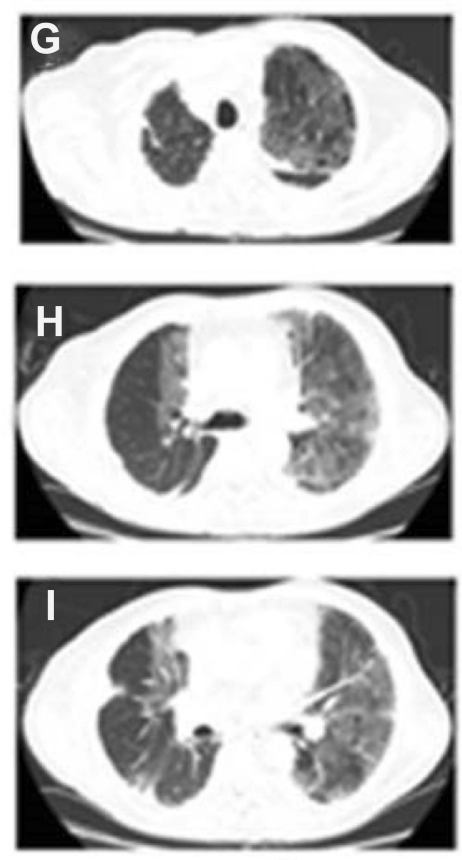

\begin{tabular}{|c|c|c|c|}
\hline PCT (ng/mL) & 0.112 & 0.131 & 0.127 \\
\hline IL-6 (pg/mL) & 65.60 & 64.37 & 105.2 \\
\hline CRP (mg/L) & 29.8 & 122 & 41.9 \\
\hline
\end{tabular}

Figure 4 A typical case of the change of cytokines and CT when developed RP. (A-C) mild interstitial changes in the left lung; (D-F), interstitial changes in both lungs; (G-I), the characteristic imaging changes of RP (small ground-glass opacity scattered in the irradiation field or long strip, large patch density enhancement shadow). Red numbers indicate the rise of the level.

Abbreviations: CT, computed tomography; IL-6, interleukin-6; CRP, C-reactive protein; PCT, procalcitonin.

essentially an aseptic inflammation, PCT levels in patients with and without RP were consistent during radiotherapy and during follow-up after radiotherapy. Single cytokine had a good ability to predict RP with the AUC of IL-6 and CRP of $0.89 \pm 0.04(95 \% \mathrm{CI}, 0.80-0.95, \mathrm{P}<0.001)$ and $0.87 \pm 0.05$ (95\% CI, 0.78-0.94, $\mathrm{P}<0.001)$ respectively. If combined IL-6 and CRP, the AUC could increase to $0.92 \pm 0.03(95 \% \mathrm{CI}$, $0.83-0.97, \mathrm{P}<0.001)$.

The incidence of RP ranges from $15 \%$ to $58 \% \%^{9,10}$ during radiotherapy or in the previous 3 months following radiotherapy. We also found similar results that the incidence of RP was $26.7 \%$. In addition, a total of $28 \mathrm{RP}$ patients, 25 of whom occurred between 6 weeks and 10 weeks. The results indicated that the period from 6 weeks to 10 weeks was the high-risk period for RP. It was necessary to be vigilant and it might be appropriate to increase the number of follow-up visits. In our study, typical RP cases showed that cytokines IL-6 and CRP could increase significantly 5 days before obvious clinical symptoms and 12 days before RP characteristic images, suggesting that they may be used as biomarkers for early detection of RP.

In addition, the clinical data of RP group and nonRP group showed $V_{20}$ and MLD were significantly different between the two groups $(\mathrm{p}<0.05)$. The study also explored that only COPD had a significant difference between the two groups, no obvious abnormality was found in smoking or chemotherapy. Laucis et al reported similar results that smoking did not increase radiation-related side effects. ${ }^{27}$ Although this study did not confirm the correlation between chemotherapy factors and RP, few studies consider that certain chemotherapy regimens and concurrent chemotherapy may increase the risk of RP. ${ }^{28}$ 


\section{Conclusions}

In conclusion, serum IL-6, CRP and PCT may predict the risk of RP during radiotherapy for patients with thoracic malignant tumors, which could provide an effective method for the early detection of RP and guide clinical practice management of RP. However, this study is only a retrospective study, and the sample size is limited. It still needs to be further validated in the prospective study.

\section{Acknowledgments}

This work was supported by Nanjing Health Science and Technology Development Project (YKK18251).

\section{Disclosure}

The authors report no conflicts of interest in this work.

\section{References}

1. Chen YY, Rubin P, Williams J, Hernady E, Smudzin T, Okunieff P. Circulating IL-6 as a predictor of radiation pneumonitis. Int J Radiat Oncol Biol Phys. 2001;49(3):641-648. doi:10.1016/S0360-3016(00) 01445-0

2. Simone CB. Thoracic radiation normal tissue injury. Semin Radiat Oncol. 2017;27(4):370-377. doi:10.1016/j.semradonc.2017.04.009

3. Gross NJ. Pulmonary effects of radiation-therapy. Ann Intern Med. 1977;86(1):81-92. doi:10.7326/0003-4819-86-1-81

4. Novakova-Jiresova A, van Luijk P, van Goor $\mathrm{H}$, Kampinga $\mathrm{HH}$, Coppes RP. Pulmonary radiation injury: identification of risk factors associated with regional hypersensitivity. Cancer Res. 2005;65 (9):3568-3576. doi:10.1158/0008-5472.CAN-04-3466

5. Chen YY, Williams J, Ding I, et al. Radiation pneumonitis and early circulatory cytokine markers. Semin Radiat Oncol. 2002;12 (1):26-33. doi:10.1053/srao.2002.31360

6. Rube CE, Palm J, Erren M, et al.. Cytokine plasma levels: reliable predictors for radiation pneumonitis?. PLOS ONE. 2008;3(8) doi:10.1371/journal.pone.0002898

7. Mehta V. Radiation pneumonitis and pulmonary fibrosis in non-smallcell lung cancer: pulmonary function, prediction, and prevention Int $J$ Radiat Oncol Biol Phys. 2005;63(1):5-24. doi:10.1016/j. ijrobp.2005.03.047

8. Marks LB, Bentzen SM, Deasy JO, et al. Radiation dose-volume effects in the lung. Int J Radiat Oncol Biol Phys. 2010;76(3):S70 S76. doi:10.1016/j.ijrobp.2009.06.091

9. Rancati T, Ceresoli GL, Gagliardi G, Schipani S, Cattaneo GM. Factors predicting radiation pneumonitis in lung cancer patients a retrospective study. Radiother Oncol. 2003;67(3):275-283. doi:10.1016/S0167-8140(03)00119-1

10. Rodrigues G, Lock M, D’Souza D, Yu E, Van Dyk J. Prediction of radiation pneumonitis by dose-volume histogram parameters in lung cancer - a systematic review. Radiother Oncol. 2004;71(2):127-138. doi:10.1016/j.radonc.2004.02.015

11. Wang DQ, Shi J, Liang SH, et al. Dose-volume histogram parameters for predicting radiation pneumonitis using receiver operating characteristic curve. Clin Transl Oncol. 2013;15(5):364-369. doi:10.1007/ s12094-012-0931-y
12. Hartsell WF, Scott CB, Dundas GS, et al. Can serum markers be used to predict acute and late toxicity in patients with lung cancer? Analysis of RTOG 91-03. Am J Clin Oncol-Cancer Clin Trials. 2007;30(4):368-376.

13. Huang YJ, Zhang WQ, Yu FR, Gao F, Cellular T. Molecular mechanism of radiation-induced lung injury. Med Sci Monit. 2017;23.

14. Anscher MS, Kong FM, Marks LB, Bentel GC, Jirtle RL. Changes in plasma transforming growth factor beta during radiotherapy and the risk of symptomatic radiation-induced pneumonitis. Int J Radiat Oncol Biol Phys. 1997;37(2):253-258. doi:10.1016/S0360-3016(96)00529-9

15. Del Giudice M, Gangestad SW, Rethinking IL. 6 and CRP: why they are more than inflammatory biomarkers, and why it matters. Brain Behav Immun. 2018;70:61-75. doi:10.1016/j.bbi.2018.02.013

16. Pfaefflin A, Schleicher E. Inflammation markers in Point-of-Care Testing (POCT). Anal Bioanal Chem. 2009;393(5):1473-1480. doi:10.1007/s00216-008-2561-3

17. Gao LQ, Liu XH, Zhang DH, et al. Early diagnosis of bacterial infection in patients with septicopyemia by laboratory analysis of PCT, CRP and IL-6. Exp Ther Med. 2017;13(6):3479-3483. doi:10.3892/etm.2017.4417

18. Yukioka H, Yoshida G, Kurita S, Kato N. Plasma procalcitonin in sepsis and organ failure. Ann Acad Med Singapore. 2001;30(5):528-531.

19. Schuetz P, Beishuizen A, Broyles M, et al. Procalcitonin (PCT)-guided antibiotic stewardship: an international experts consensus on optimized clinical use. Clin Chem Lab Med. 2019;57 (9):1308-1318. doi:10.1515/cclm-2018-1181

20. Sakr Y, Sponhoz C, Tuche F, Brunkhorst F, Reinhart K. The role of procalcitonin in febrile neutropenic patients: review of the literature. Infection. 2008;36(5):396-407. doi:10.1007/s15010-008-7374-y

21. Sanuki N, Ono A, Komatsu E, et al. Association of computed tomography-detected pulmonary interstitial changes with severe radiation pneumonitis for patients treated with thoracic radiotherapy. J Radiat Res. 2012;53(1):110-116. doi:10.1269/jrr.110142

22. Fu -Z-Z, Peng Y, Cao L-Y, Chen Y-S, Li K, Fu B-H. Correlations between serum IL-6 levels and radiation pneumonitis in lung cancer patients: a meta-analysis. J Clin Lab Anal. 2016;30(2):145-154. doi:10.1002/jcla.21828

23. Sakai M, Iwakawa M, Iwakura Y, Ohta T, Tsujii H, Imai T. CD44 and Bak expression in IL-6 or TNF-alpha gene knockout mice after whole lung irradiation. J Radiat Res. 2008;49(4):409-416. doi:10.1269/ jrr.07087

24. Stenmark MH, Cai XW, Shedden K, et al. Combining physical and biologic parameters to predict radiation-induced lung toxicity in patients with non-small-cell lung cancer treated with definitive radiation therapy. Int J Radiat Oncol Biol Phys. 2012;84(2):E217-E222. doi:10.1016/j.ijrobp.2012.03.067

25. Hart JP, Broadwater G, Rabbani Z, et al. Cytokine profiling for prediction of symptomatic radiation-induced lung injury. Int J Radiat Oncol Biol Phys. 2005;63(5):1448-1454. doi:10.1016/j.ijrobp.2005.05.032

26. Gelalis ID, Arnaoutoglou CM, Politis AN, Batzaleksis NA, Katonis PG, Xenakis TA. Bacterial wound contamination during simple and complex spinal procedures. a prospective clinical study. Spine J. 2011;11(11):1042-1048.

27. Laucis AM, Sun Y, Schipper M, et al. Active smoking is not associated with increased radiation-induced toxicity in locally advanced lung cancer patients. Int J Radiat Oncol Biol Phys. 2018;102(3): S231-S231. doi:10.1016/j.ijrobp.2018.07.166

28. McCurdy M, McAleer MF, Wei W, et al. Induction and concurrent taxanes enhance both the pulmonary metabolic radiation response and the radiation pneumonitis response in patients with esophagus cancer. Int $J$ Radiat Oncol Biol Phys. 2010;76(3):816-823. doi:10.1016/j.ijrobp.2009.02.059 


\section{Publish your work in this journal}

Cancer Management and Research is an international, peer-reviewed The manuscript management system is completely online and includes open access journal focusing on cancer research and the optimal use of a very quick and fair peer-review system, which is all easy to use. preventative and integrated treatment interventions to achieve improved Visit http://www.dovepress.com/testimonials.php to read real quotes outcomes, enhanced survival and quality of life for the cancer patient.

from published authors. 